\title{
Correction to: A Prospective Cohort Study on Serum Sodium and Clinical Outcome in Pediatric Nontraumatic Coma
}

\author{
Akanksha Gupta ${ }^{1} \cdot$ Ramachandran Rameshkumar $^{1} \cdot$ Muthu Chidambaram ${ }^{1} \cdot$ Tamil Selvan $^{1}$. \\ Subramanian Mahadevan ${ }^{1}$
}

Published online: 29 September 2021

(c) Dr. K C Chaudhuri Foundation 2021

\section{Correction to: Indian Journal of Pediatrics https://doi.org/10.1007/s12098-021-03726-4}

Figures 1 and 2 are not related to the published version of the article. The correct images are shown below.

The original article has been corrected.

The original article can be found online at https://doi.org/10.1007/ s12098-021-03726-4.

Ramachandran Rameshkumar

krramesh_iway@yahoo.co.in

1 Department of Pediatrics, Division of Pediatric Critical Care, Jawaharlal Institute of Postgraduate Medical Education and Research (JIPMER), Puducherry 605006, India 


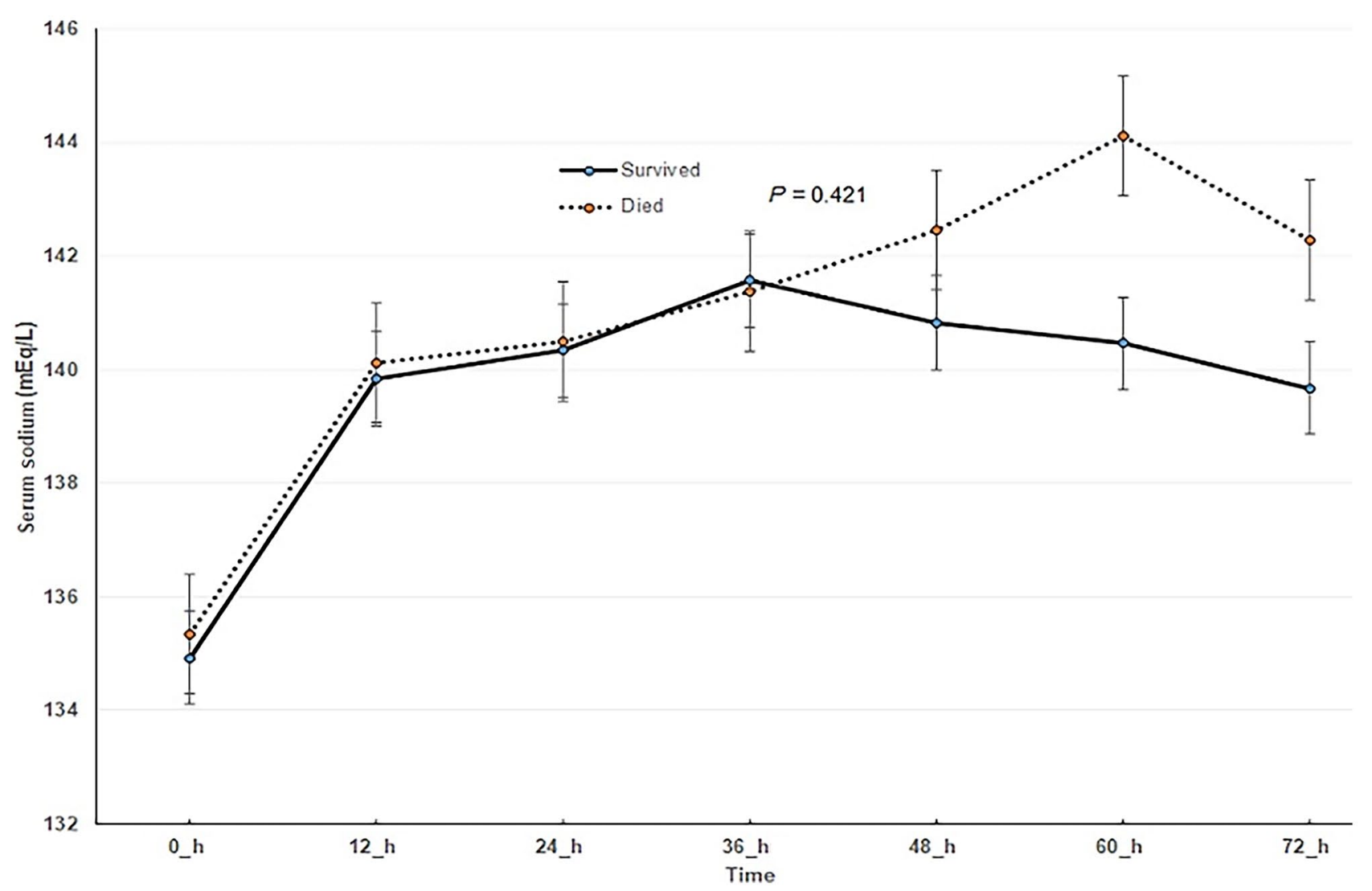

Fig. 1 Trend of serum sodium (mEq/L) in the first $72 \mathrm{~h}$ 


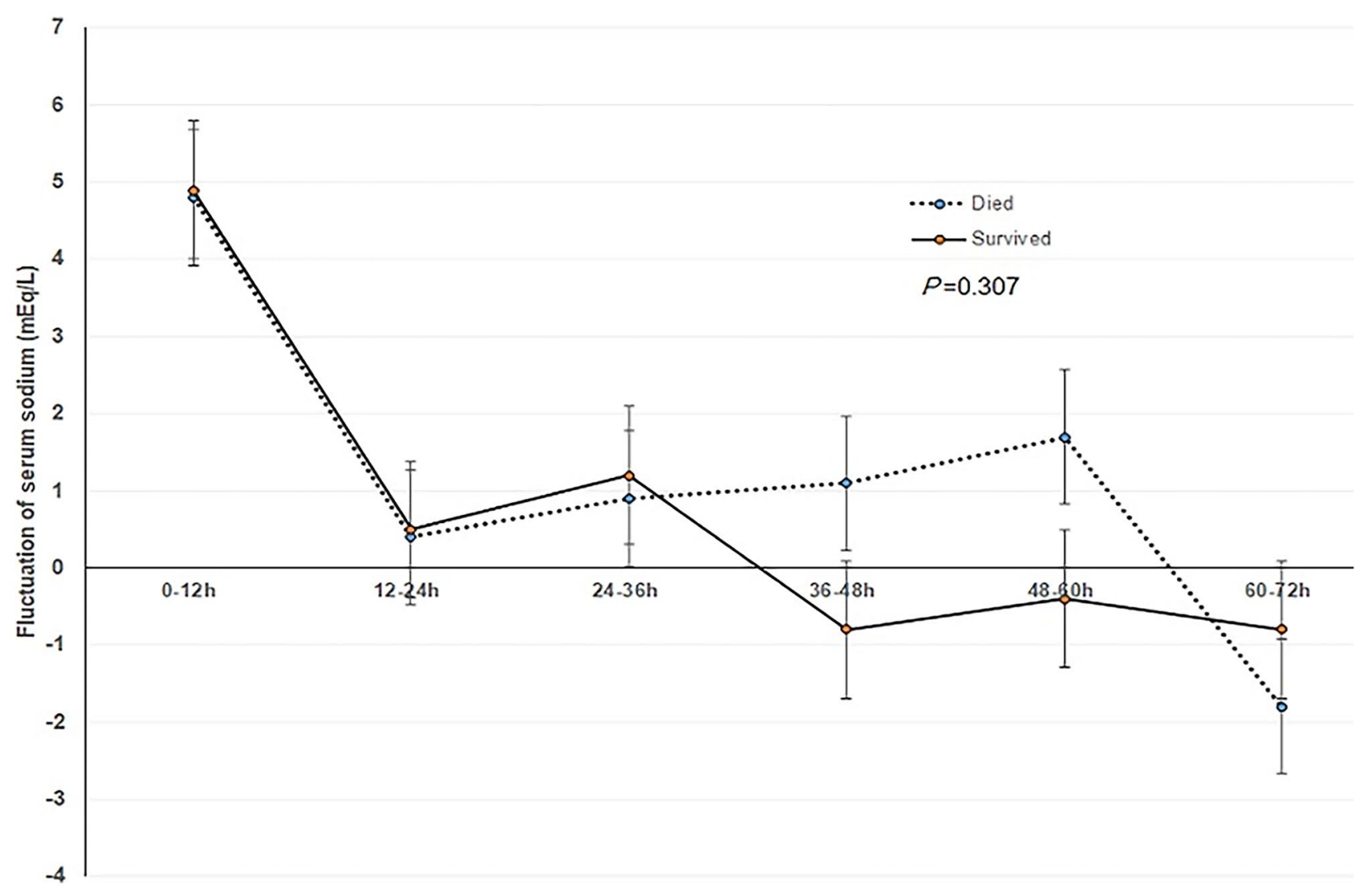

Fig. 2 The fluctuation of serum sodium (mEq/L) in the first $72 \mathrm{~h}$

Publisher's Note Springer Nature remains neutral with regard to jurisdictional claims in published maps and institutional affiliations. 